\title{
Synthesis and Characterization of ZnTe Hierarchical Nanostructures
}

\author{
Baohua Zhang, Fuqiang Guo, and Wei Wang \\ Department of Physics, Changji College, Changji 831100, China \\ Correspondence should be addressed to Fuqiang Guo, flowerforever@yahoo.cn \\ Received 20 September 2011; Revised 14 November 2011; Accepted 14 November 2011 \\ Academic Editor: William W. Yu \\ Copyright ( 2012 Baohua Zhang et al. This is an open access article distributed under the Creative Commons Attribution License, \\ which permits unrestricted use, distribution, and reproduction in any medium, provided the original work is properly cited.
}

Single-crystalline ZnTe hierarchical nanostructures have been successfully synthesized by a simple thermal evaporation technology. The as-prepared products were characterized with X-ray diffraction (XRD), scanning electron microcopy (SEM), transmission electron microscope (TEM), and photoluminescence spectrum (PL). These results showed that the ZnTe hierarchical nanostructures consisted of nanowires and nanolumps. The room temperature PL spectrum exhibited a pure green luminescence centered at $545 \mathrm{~nm}$. The growth mechanism of hierarchical nanostructure was also discussed.

\section{Introduction}

As an important member of groups II-VI semiconductors, ZnTe (band gap 2.26 eV) [1] has attracted much attention due to outstanding optoelectronic and thermoelectric properties $[2,3]$, and it is now widely used for nanometerscale electronics and optoelectronics [4-9]. Several methods have been explored to grow ZnTe-based nanostructures [1012]. $\mathrm{Li}[13]$ and coworkers reported that the semiconductor ZnTe nanowire arrays have been synthesized by the pulsed electrochemical deposition from aqueous solutions into porous anodic alumina membranes. Jun [14] and coworkers reported that the synthesis of spherical and rodlike nanocrystals using a single molecular precursor ([ $\mathrm{Zn}$ $\left.(\mathrm{TePh})_{2}\right][$ TMEDA]). Recently, the synthesis of $\mathrm{ZnTe} / \mathrm{CdSe}$ core/shell spherical NCs with varied core sizes and shell thicknesses, as well as their widely tuned emission from the visible to near-infrared regions, was reported by Xie' and coworkers [15]. Conversely, ZnTe nanorods were preferentially synthesized using autoclave reactions [16, 17]. Compared with the sample nanostructures, the hierarchical nanostructures have attracted a great deal of attentions over the past few years due to their improved light absorption efficiency and carrier transport. However, the ZnTe with hierarchical nanostructures has not been reported so far.
In this paper, highly crystallized ZnTe hierarchical nanostructures are synthesized on graphite wafers using a simple thermal evaporation technology. The as-prepared samples are characterized by scanning electron microcopy (SEM), transmission electron microscopy (TEM), and X-ray diffraction (XRD). These results showed that the ZnTe hierarchical nanostructures consist of nanowires and nanolumps. The possible growth mechanism has also been discussed.

\section{Experimental Section}

The thermal evaporation apparatus for sample fabrication is illustrated in Figure 1.

Source materials of high pure ZnTe (99.99\%) powder were placed in an alumina boat which is in the heating center of a horizontal alumina tube furnace. After a piece of graphite wafer was cleaned in piranha solution $(30 \%$ $\mathrm{H}_{2} \mathrm{O}_{2} / 20 \% \mathrm{H}_{2} \mathrm{SO}_{4}$ ) and rinsed with deionized water, it was placed downstream to act as deposition substrates for materials growth. The distance between the graphite substrate and the source material is $15 \mathrm{~cm}$. Prior to heating, the system was evacuated and flushed with high pure Ar for $1 \mathrm{~h}$ to eliminate oxygen. Then the furnace was heated to $1000^{\circ} \mathrm{C}$ in $120 \mathrm{~min}$ and held at this temperature for 


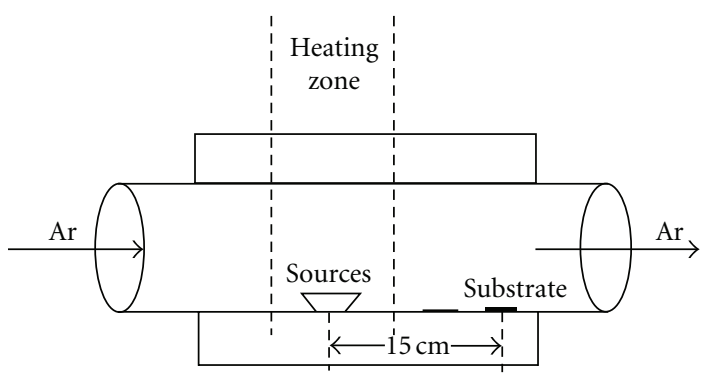

FIgURE 1: Schematic illustration of the furnace used in the experiment.

60 minutes, and subsequently cooled to room temperature under a constant flow rate of 300 SCCM (standard cubic centimeters/minute) Ar.

A Philips XL 30 FEG scanning electron microscope (SEM) with an energy-dispersive X-ray spectroscope (EDS) was used to observe the morphologies and elemental compositions of the samples. An X-ray diffractometer (XRD) (Japan Mac science) with $\mathrm{Cu} \mathrm{K} \alpha$ radiation was used to obtain phase compositions of the samples. A JEOL 2010 transmission electron microscope (TEM) with selected-area electron diffraction (SAED) was used to analyze the morphology and microstructure. A Hitachi F-7000FL spectrophotometer was used to measure the room-temperature photoluminescence (PL).

\section{Results and Discussion}

The large amount of hierarchical nanostructures obtained in such experiments is demonstrated in Figure 2(a). It indicates that high-density nanowires are grown homogeneously on a large area of the graphite wafer. The diameters of the main nanowires range from 300 to $500 \mathrm{~nm}$, and their lengths are in the range of several ten micrometers. The SEM images of the typical 1-fold nanostructure where the nanoparticles grow on one side of the primary nanowires are shown in Figures 2(b) and 2(c). It can be clearly observed that the nanowires are lumpy on the surface, and the nanoparticles have diameter of about 200-1000 nm. The corresponding EDS spectrum inserted in Figure 2(b) indicates that the hierarchical nanostructure is composed of $51 \% \mathrm{Zn}$ and $49 \%$ Te atoms, close to $1: 1$ stoichiometry of ZnTe. The crystal structure and phase composition of the obtained products were characterized by powder X-ray diffraction (XRD) in Figure 2(d). All the diffraction peaks match well with the standard powder diffraction data (JCPDS no. 65-0385), the four strongest peaks of the product could be indexed to the (111), (200), (220), and (311) planes of the cubic zincblende-structured ZnTe with a lattice constant of $a=6.10 \AA$. $\mathrm{XRD}$ diffraction peaks from $\mathrm{Te}, \mathrm{Zn}$, or other impurities are not observed in the products.

The morphology and microstructure of the products are further checked using TEM. Figure 3(a) is the morphology TEM image of the lumpy nanowires. It can be seen that a typical feature of ZnTe hierarchical nanowire consists of nanowires and nanolumps. The nanowires have a diameter of about 200-250 nm, and the height of the "lumpy hills" ranges from 100 to $300 \mathrm{~nm}$. The area marked with 1 and 2 is magnified to get the high-resolution TEM images, as shown in Figures 3(b) and 3(c), respectively. It is shown that both the trunk and the edge have the uniform crystal structure, showing the lattice fringes of the $\{-220\}$ and $\{-1-11\}$ planes with a d spacing of $0.21 \mathrm{~nm}$ and $0.35 \mathrm{~nm}$, respectively. Electron diffraction (Figure $3(\mathrm{~d})$ ) shows that the growth direction of the $\mathrm{ZnTe}$ hierarchical nanowire is [-220].

Vapor-solid (VS) and vapor-liquid-solid (VLS) mechanisms have been widely used to explain the formation of onedimensional structures $[18,19]$. In our work, we considered that the formation of $\mathrm{ZnTe}$ hierarchical structures could be explained by the VS mechanism. The process is shown in Figure 4, and four steps are present. Firstly, with the increasing temperature of source, the ZnTe powders start evaporating. Secondly, the $\mathrm{Zn}$ and $\mathrm{Te}_{2}$ gas flows with the Ar carrier gas to the low-temperature area, and react with each other. The ZnTe nanowires grew by means of a selfcatalyst vapor solid (VS) mechanism. Thirdly, when the temperature is lower, some $\mathrm{ZnTe}$ nanodroplets are formed in the atmosphere and adhered to the surface of newly formed $\mathrm{ZnTe}$ nanowires. Finally, $\mathrm{Zn}$ and $\mathrm{Te}_{2}$ vapor species dissolve in ZnTe droplets continually and ZnTe hierarchical structures were obtained. These chemical processes are expressed as (1), (2), and (3):

$$
\begin{gathered}
\mathrm{ZnTe}(\mathrm{s}) \longrightarrow \mathrm{ZnTe}(\mathrm{g}), \\
2 \mathrm{ZnTe}(\mathrm{g}) \longrightarrow 2 \mathrm{Zn}(\mathrm{g})+\mathrm{Te}_{2}(\mathrm{~g}), \\
2 \mathrm{Zn}(\mathrm{g})+\mathrm{Te}_{2} \longrightarrow 2 \mathrm{ZnTe}(\mathrm{s}) .
\end{gathered}
$$

Room-temperature PL properties of the ZnTe hierarchical structure was also investigated using a He-Cd laser line at $325 \mathrm{~nm}$ as the excitation source (Figure 5). Previous reports have shown that the PL properties of $\mathrm{ZnTe}$ are sensitive to the morphologies which are influenced by synthetic conditions. For example, Tooru and coworkers reported that PL spectra of ZnTe homoepitaxial layers exhibited a sharp excitonic emission at $2.375 \mathrm{eV}$ [20]. In our experiment, the spectrum exhibits a green emission centered at $545 \mathrm{~nm}(2.277 \mathrm{eV})$, which is higher than the band gap of bulk Zinc blende ( $\mathrm{Eg}=$ $2.26 \mathrm{eV}$ ). We believe that the small blue shift of the PL peak is caused by anti-Stoke's shift.

\section{Conclusion}

In summary, ZnTe hierarchical structures were successfully synthesized via a simple thermal evaporation technology. SEM, EDS, and TEM show that the ZnTe hierarchical nanostructures consist of nanowires and nanolumps. Vapor-solid (VS) growth mechanism was proposed for the formation of ZnTe hierarchical structures. Photoluminescence (PL) measurements at room temperature also demonstrate that the synthesized ZnTe hierarchical structures emit a strong 


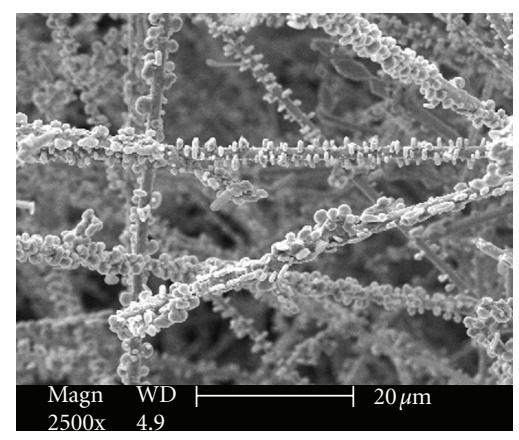

(a)

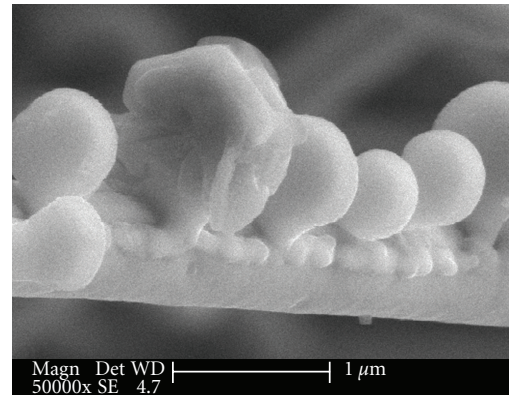

(c)

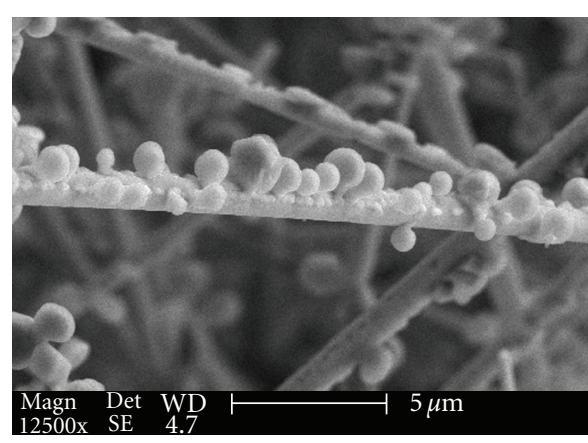

(b)

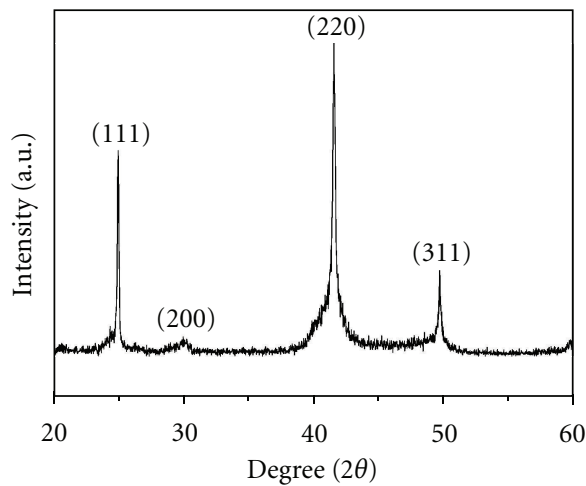

(d)

FIGURE 2: Low magnification (a) and high magnification and SEM images and corresponding EDS spectrum of the sample (b and c), and XRD spectrum of the sample $(\mathrm{d})$.

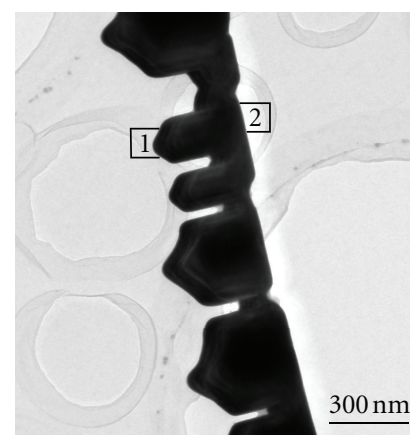

(a)

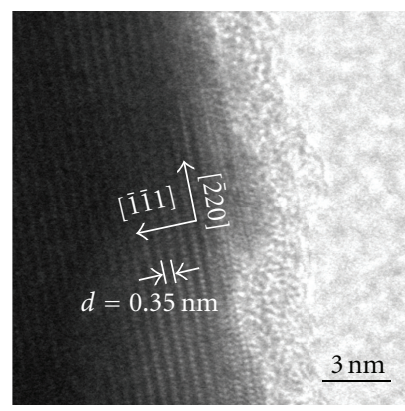

(c)

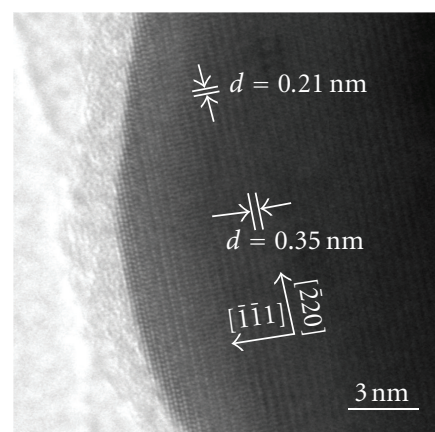

(b)

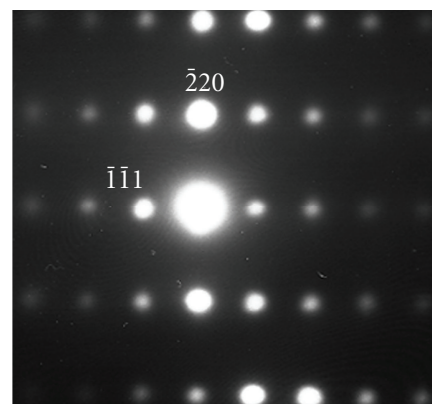

(d)

Figure 3: TEM image of an individual ZnTe nanowire (a and b), HRTEM image (c), and SAED pattern (d). 

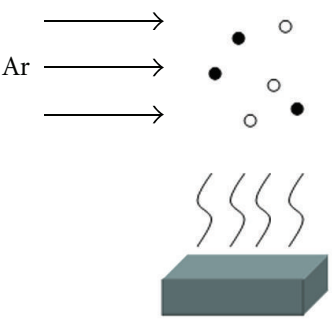

(a)
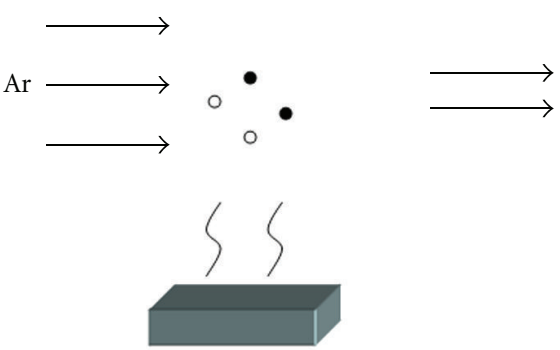

(c)
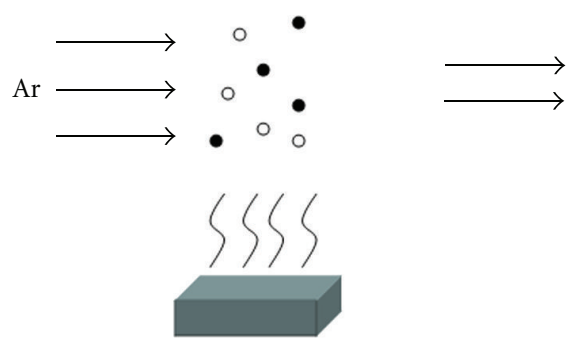

0

$\circ$
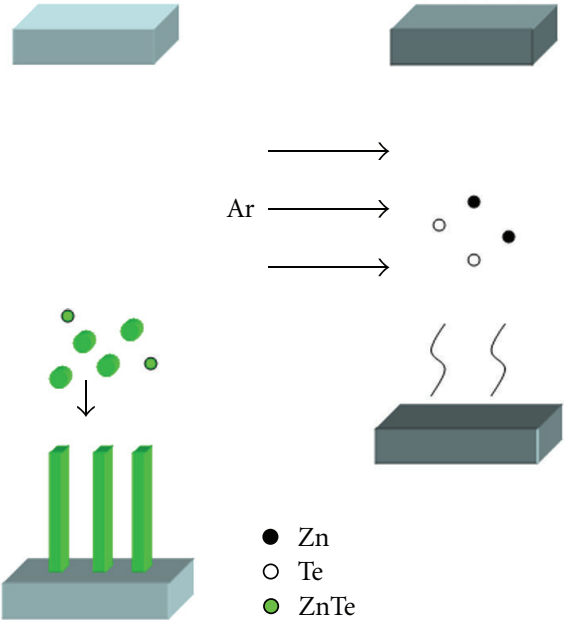

- $\mathrm{Zn}$

○ $\mathrm{Te}$

- $\mathrm{ZnTe}$ (b)
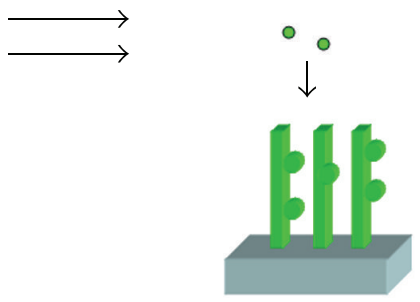

FIGURE 4: Schematic illustration of the formation process of ZnTe hierarchical nanostructures.

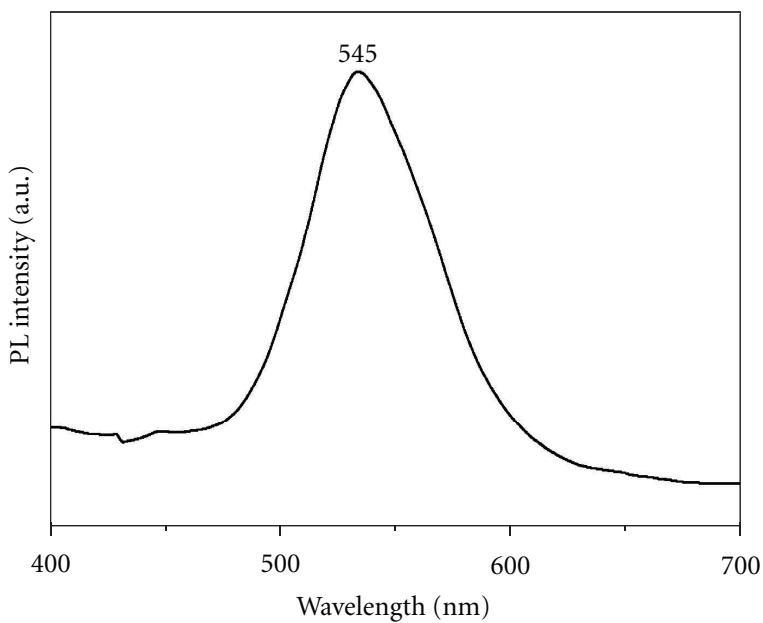

Figure 5: PL spectrum of the nanowire arrays at an excitement wavelength of $325 \mathrm{~nm}$.

and stable green light and that their luminescent peak is at about $545 \mathrm{~nm}$.

\section{Acknowledgment}

This work is supported finantially by Scientific Research Program of the Higher Education Institution of Xinjiang (XJEDU2010S46).

\section{References}

[1] K. Yoshino, M. Yoneta, T. Yabe, K. Ohmori, H. Saito, and M. Ohisihi, "Optical and electrical characterization of highquality P-doped ZnTe substrates," Physica B, vol. 340-342, pp. 254-257, 2003.

[2] N. Mingo, "Thermoelectric figure of merit of II-VI semiconductor nanowires," Applied Physics Letters, vol. 85, no. 24, pp. 5986-5988, 2004.

[3] B. Wan, C. Hu, B. Feng, J. Xu, Y. Zhang, and Y. Tian, "Optical properties of ZnTe nanorods synthesized via a facile low-temperature solvothermal route," Materials Science and Engineering B, vol. 171, no. 1-3, pp. 11-15, 2010.

[4] J. A. Zapien, Y. Jiang, X. M. Meng et al., "Room-temperature single nanoribbon lasers," Applied Physics Letters, vol. 84, no. 7, pp. 1189-1191, 2004.

[5] M. S. Gudiksen, L. J. Lauhon, J. Wang, D. C. Smith, and C. M. Lieber, "Growth of nanowire superlattice structures for nanoscale photonics and electronics," Nature, vol. 415, no. 6872, pp. 617-620, 2002.

[6] J. C. Johnson, H. Yan, P. Yang, and R. J. Saykally, "Optical cavity effects in $\mathrm{ZnO}$ nanowire lasers and waveguides," Journal of Physical Chemistry B, vol. 107, no. 34, pp. 8816-8828, 2003.

[7] W. Zhou, D. Tang, A. Pan, Q. Zhang, Q. Wan, and B. Zou, "Structure and photoluminescence of pure and indium-doped ZnTe microstructures," Journal of Physical Chemistry C, vol. 115, no. 5, pp. 1415-1421, 2011.

[8] H. Yan, J. Johnson, M. Law et al., "ZnO nanoribbon microcavity lasers," Advanced Materials, vol. 15, no. 22, pp. 1907-1911, 2003.

[9] J. C. Johnson, K. P. Knutsen, H. Yan et al., "Ultrafast carrier dynamics in single $\mathrm{ZnO}$ nanowire and nanoribbon lasers," Nano Letters, vol. 4, no. 2, pp. 197-204, 2004. 
[10] F. Wang, A. Dong, J. Sun, R. Tang, H. Yu, and W. E. Buhro, "Solution-liquid-solid growth of semiconductor nanowires," Inorganic Chemistry, vol. 45, no. 19, pp. 7511-7521, 2006.

[11] Y. W. Jun, J. E. Koo, and J. Cheon, "One-step synthesis of size tuned zinc selenide quantum dots via a temperature controlled molecular precursor approach," Chemical Communications, no. 14, pp. 1243-1244, 2000.

[12] Y. Xia, P. Yang, Y. Sun et al., "One-dimensional nanostructures: synthesis, characterization, and applications," Advanced Materials, vol. 15, no. 5, pp. 353-389, 2003.

[13] L. Li, Y. Yang, X. Huang, G. Li, and L. Zhang, "Fabrication and characterization of single-crystalline ZnTe nanowire arrays," Journal of Physical Chemistry B, vol. 109, no. 25, pp. 1239412398, 2005.

[14] Y. W. Jun, C. S. Choi, and J. Cheon, "Size and shape controlled ZnTe nanocrystals with quantum confinement effect," Chemical Communications, no. 1, pp. 101-102, 2001.

[15] R. Xie, X. Zhong, and T. Basche, "Synthesis, characterization, and spectroscopy of type-II core/shell semiconductor nanocrystals with ZnTe cores," Advanced Materials, vol. 17, no. 22, pp. 2741-2745, 2005.

[16] Y. Li, Y. Ding, and Z. Wang, "A novel chemical route to ZnTe semiconductor nanorods," Advanced Materials, vol. 11, no. 10, pp. 847-850, 1999.

[17] J. Du, L. Xu, G. Zou, L. Chai, and Y. Qian, "Solvothermal synthesis of single crystalline ZnTe nanorod bundles in a mixed solvent of ethylenediamine and hydrazine hydrate," Journal of Crystal Growth, vol. 291, no. 1, pp. 183-186, 2006.

[18] R. S. Wagner and W. C. Ellis, "Vapor-liquid-solid mechanism of single crystal growth," Applied Physics Letters, vol. 4, no. 5, pp. 89-90, 1964.

[19] X. Duan and C. M. Lieber, "Laser-assisted catalytic growth of single crystal GaN nanowires," Journal of the American Chemical Society, vol. 122, no. 1, pp. 188-189, 2000.

[20] T. Tanaka, K. Hayashida, S. Wang, Q. Guo, M. Nishio, and H. Ogawa, "Photoluminescence properties of ZnTe homoepitaxial layers grown by synchrotron-radiation-excited growth using nitrogen carrier gas," Nuclear Instruments and Methods in Physics Research, Section B, vol. 199, pp. 356-360, 2003. 

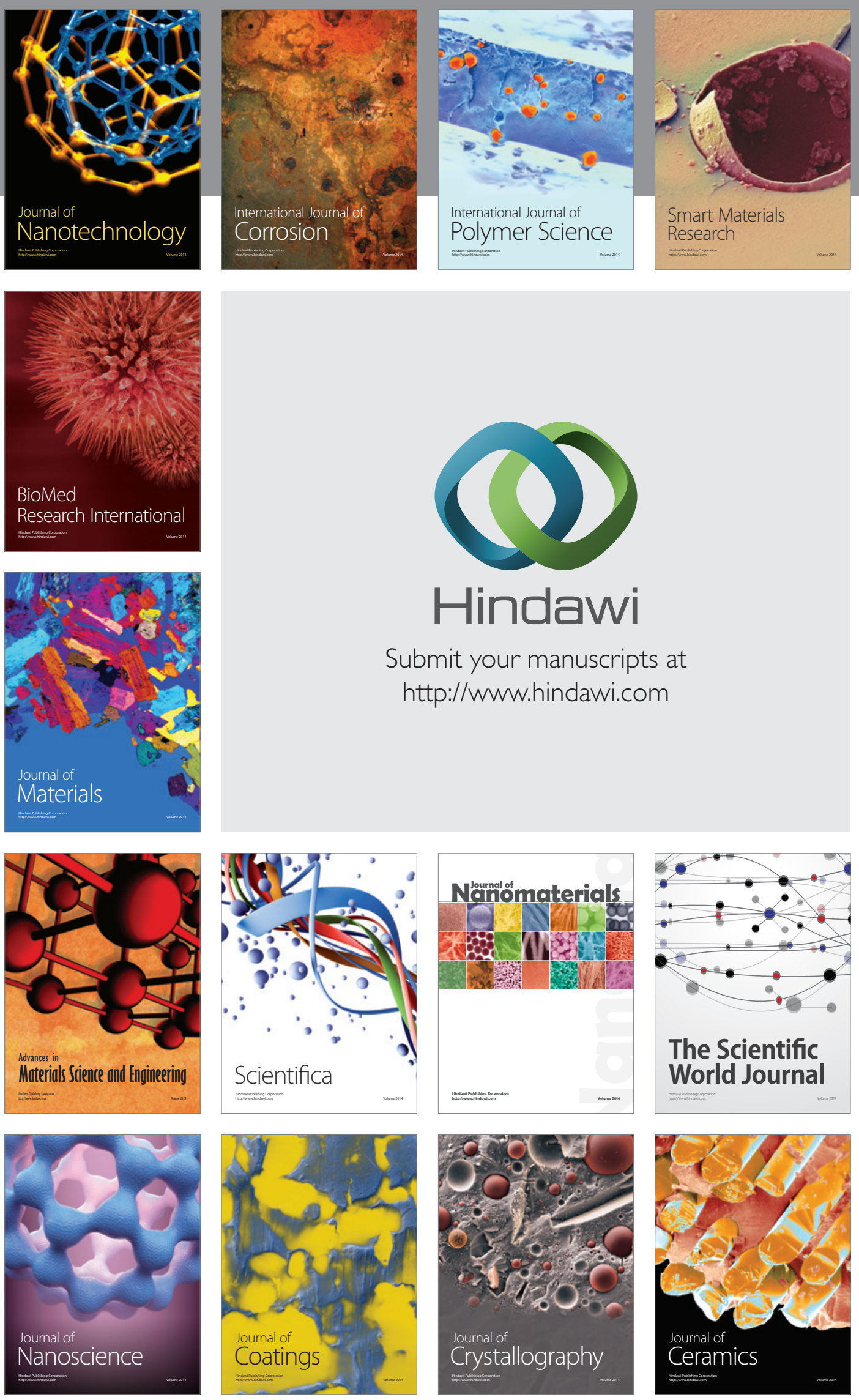

The Scientific World Journal

Submit your manuscripts at

http://www.hindawi.com

\section{World Journal}

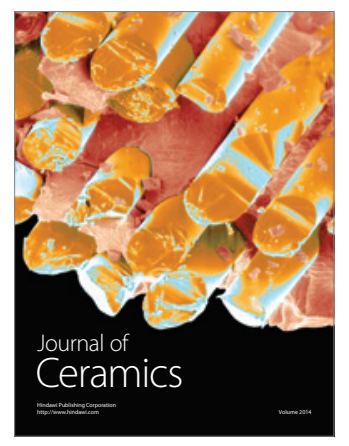

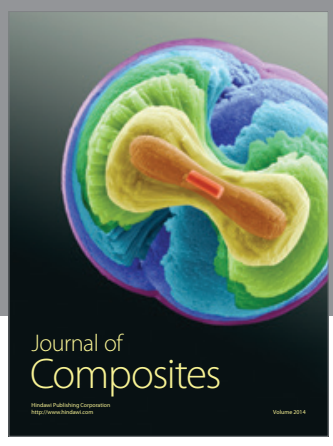
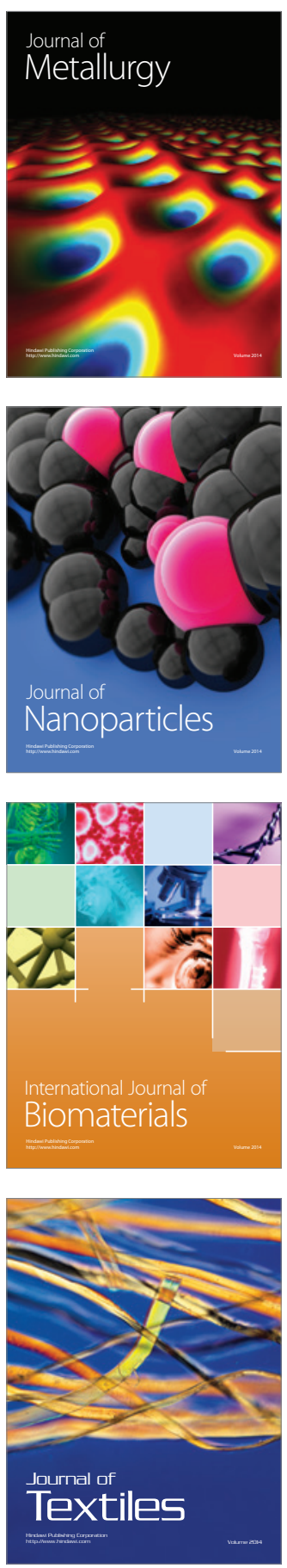